\title{
Philosophiques
}

\section{Du Discours sur l'Inégalité à l'Émile : le parcours anthropologique de Rousseau}

\section{Vinh-De Nguyen}

Volume 15, numéro 2, automne 1988

URI : https://id.erudit.org/iderudit/027053ar

DOI : https://doi.org/10.7202/027053ar

Aller au sommaire du numéro

Éditeur(s)

Société de philosophie du Québec

ISSN

0316-2923 (imprimé)

1492-1391 (numérique)

Découvrir la revue

Citer cet article

Nguyen, V.-D. (1988). Du Discours sur l'Inégalité à l'Émile : le parcours anthropologique de Rousseau. Philosophiques, 15(2), 405-419.

https://doi.org/10.7202/027053ar
Résumé de l'article

Le point de départ de cette étude est un passage de la préface du Discours sur l'Inégalité, passage qui, quoique souligné par Rousseau, n'a pas longtemps retenu l'attention des commentateurs. Il s'agit du texte suivant : « ... une bonne solution du problème suivant ne me paraîtrait pas indigne des Aristotes et des Plines de notre siècle : Quelles expériences seraient nécessaires pour parvenir à connaître l'homme naturel? et quels sont les moyens de faire ces expériences au sein de la société? ». La lecture du Manuscrit Favre, où l'on retrouve les mêmes interrogations, nous autorise à voir dans l'Emile la solution de Rousseau au problème posé et à mieux saisir les limites du Second Discours en tant que discours anthropologique. 


\title{
DU DISCOURS SUR L'INEGALITÉ A L'EMILE : LE PARCOURS ANTHROPOLOGIQUE DE ROUSSEAU
}

\author{
par Vinh-De Nguyen
}

\begin{abstract}
RÉSUMÉ. Le point de départ de cette étude est un passage de la préface du Discours sur l'Inégalité, passage qui, quoique souligné par Rousseau, n'a pas longtemps retenu l'attention des commentateurs. Il s'agit du texte suivant : «... une bonne solution du problème suivant ne me paraîtrait pas indigne des Aristotes et des Plines de notre siècle: Quelles expériences seraient nécessaires pour parvenir à connaitre l'homme naturel? et quels sont les moyens de faire ces expériences au sein de la société?"'.

La lecture du Manuscrit Favre, où l'on retrouve les mêmes interrogations, nous autorise à voir dans l'Émile la solution de Rousseau au problème posé et à mieux saisir les limites du Second Discours en tant que discours anthropologique.
\end{abstract}

ABSTRACT. This study is a commentary on a passage of the preface of Rousseau's Discourse on Inequality: «It appears to me that a good solution of the following problem would be not unworthy of the Aristotles and Plinys of the present age: What experiments would have to be made, to discover the natural man? And how are those experiments to be made in a state of society?

The "Manuscrit Favre», in which Rousseau formulates the same questions, allows us to state that the author sets forth his solution in his Emile, and to perceive the limits of the Second Discourse as an anthropological work.

Les commentateurs n'attribuent pas la même signification au Discours sur l'Inégalité de Rousseau. Si Jean Starobinski y trouve une théodicée ${ }^{1}$, Victor Goldschmidt, percevant l'intention de

1. “Le second Discours est une théodicée " (Jean Starobinski, Jean-Jacques Rousseau, la transparence et l'obstacle, Gallimard, Paris, 1971, p. 346). 
l'auteur de rivaliser avec les jusnaturalistes, le considère comme un “ouvrage de droit naturel» ${ }^{2}$, alors qu'aux yeux de Jean-Louis Lecercle ce discours est essentiellement une œuvre politique 3. Mais si les interprétations peuvent diverger sur la signification dernière du second Discours, les commentateurs s'accordent tous pour reconnaittre dans cette œuvre un discours anthropologique, car l'intention de faire une étude de la nature humaine est précisée au début de l'Exorde ${ }^{4}$.

Que le Discours doive commencer par cette étude se justifie non seulement par sa pertinence à l'égard de toute tentative de répondre à la question posée par l'Académie de Dijon, mais encore par son importance dans le traitement que Rousseau entend donner aux problèmes sociaux et politiques : L'« étude de l'homme originel..., lit-on dans la préface, est... le seul bon moyen qu'on puisse employer pour lever ces foules de difficultés qui se présentent sur l'origine de l'inégalité morale, sur les vrais fondements du corps politique, sur les droits réciproques de ses membres, et sur mille autres questions semblables, aussi importantes que mal éclaircies ${ }^{5}$.

L'étude de la nature humaine, occupant toute la première partie du Discours sur l'Inégalité, se présente comme la description d'un état de nature où l'homme originel vivrait «errant dans les forêts sans industries, sans parole, sans domicile, sans guerre, et sans liaisons, sans nul besoin de ses semblables... et se suffisant à lui-même... " ${ }^{6}$.

Avons-nous là toute la pensée anthropologique de Rousseau ? Pouvons-nous voir dans ce portrait de l'« homme sauvage » la voie qui permettrait, selon le vœu de l'auteur, de «lever ces foules de difficultés » dont il parle?

2. «... le second Discours en dépit des apparences, n'est pas un récit historique... mais un ouvrage de droit naturel..." (Victor GolDsCHMIDT, Anthropologie et politique, les principes du système de Rousseau, Vrin, Paris, 1974, p. 639).

3. «Le premier Discours était un traité de morale. Le deuxième est une ouvre politique, qui place d'emblée Rousseau dans la lignée des grands penseurs politiques » (JeanLouis LECERCLE, Introduction à J.J. Rousseau, De l'inégalité parmi les bommes, Éd. sociales, Paris, 1965, p. 31).

4. “C'est de l'homme que j'ai à parler" (CEuvres complètes, Pléiade, III, p. 131).

5. Ibid., p. 126.

6. Ibid., p. 159-160 
On ne saurait répondre à ces questions de manière satisfaisante tant qu'on ne connaît pas le programme de recherche anthropologique de l'auteur de l'Émile, et qu'on ne sait pas dans quelle mesure le second Discours le réalise.

Ce qui s'est d'abord imposé à Rousseau au début de sa recherche, c'est l'opposition entre l'homme «tel que l'a formé la nature ${ }^{7}$ et l'homme tel qu'il est actuellement à la suite des changements que "la succession des temps et des choses a dû produire dans sa constitution originelle ${ }^{9}$. Cette opposition dicte la direction à suivre dans l'étude de la nature humaine : il s'agit de "démêler ce qu'il y a d'originaire et d'artificiel dans la nature actuelle de l'homme " ${ }^{9}$, de retrouver l'état de nature en deçà de tous les ajouts dus aux circonstances et aux progrès que l'homme a apportés à son " état primitif ». Mais comment démêler ? Comment espérer retrouver la "constitution originelle " de l'âme humaine, défigurée comme elle est par "mille causes sans cesse renaissantes » au sein de la société, par l' « acquisition d'une multitude de connaissances et d'erreurs » et par le «choc continuel des passions » ${ }^{10}$ ?

L'idée de séparer l'artificiel et le naturel n'est nullement propre à Rousseau. Pour Locke, Buffon et autres penseurs de l'époque, l'observation d'un état de nature peut se faire au moyen d'une expérimentation ${ }^{11}$. Rousseau est loin de repousser une telle idée; on le voit au contraire, proposer aux hommes compétents le problème de la différenciation expérimentale du naturel et de l'artificiel : «quelles expériences seraient nécessaires pour parvenir à connaître l'homme naturel; et quels sont les moyens de faire ces expériences au sein de la société ? "12

Quoiqu'il estime la démarche expérimentale pertinente, Rousseau ne l'a pourtant pas adoptée dans son Discours. Il s'est donné une autre méthode, qui s'oppose aux deux méthodes analytique et empirique. La première, pratiquée par Hobbes méconnaît la

7. lbid., p. 122.

8. Id.

9. Euvres complètes, Pléiade, III, p. 123.

10. Ibid., p. 122.

11. Sur ce sujet voir la note de Jean Starobinski dans Euvres complètes, P1. III, p. 1295, note p. 124. Voir aussi René Pomeau, « De la nature : essai sur la vie littéraire d'une idée » dans Revue de l'enseignement supérieur (1959), $\mathrm{n}^{\circ} 1$ janvier-mars, p. 114-116.

12. Guvres complètes, P1. III, P. 123-124 
dimension historique des faits humains, confondant ainsi le social avec le naturel ${ }^{13}$. La deuxième a le défaut de ne pas convenir à une recherche qui vise plus «à éclaircir, la nature des choses qu'à montrer la véritable origine ${ }^{14}$. Sa méthode consiste à faire abstraction chez l'être humain de tout ce qui paraît dû à de « longs progrès». Ce «déshabillage de l'esprit» (Jean Starobinski) est commun à bien des auteurs de l'époque; ce qui rend l'approche de Rousseau originale, c'est, comme l'a bien montré Jean Starobinski, le fait d'avoir tenu compte des "paramètres temporels et collectifs ${ }^{15}$. Cette méthode s'exerce sur la base de deux postulats: la fertilité de la nature et la dispersion des hommes ${ }^{16}$.

Il n'entre pas dans notre propos de suivre l'auteur dans l'application de sa méthode, mais de chercher à savoir si la description de l'«homme sauvage» épuise toute sa pensée anthropologique.

Plusieurs déclarations de Rousseau nous empêchent de voir dans le second Discours un exposé définitif de sa conception de l'homme ${ }^{17}$. Tout d'abord, il affirme dans la préface qu'il n'entreprend pas de résoudre le problème de la connaissance du naturel et de l'artificiel ${ }^{18}$. Conscient des difficultés qui attendent tous ceux qui

13. «... pour le (l'homme) bien connaître, il faut savoir démêler la gradation naturelle de ses sentiments et ce n'est point chez les habitants d'une grande ville qu' il faut chercher le premier trait de la nature dans l'empreinte du caeur humain. Ainsi, cette méthode analytique n'offre-t-elle qu'abîmes et mystères, où le plus sage comprend le moins ». (Fragment sur l'État de guerre, OEuvres complètes, P1., III, p. 612).

14. CEuvres complètes, P1., III, p. 133.

15. Jean Starobinski, Introduction au Discours sur l'Inégalité, CEuvres complètes, P1. III, p. LV. Ce texte est repris dans Jean STAROBINSKI, Jean-Jacques Rousseau, la transparence et l'obstacle, Gallimard, Paris, 1971, p. 342.

16. "La Terre abandonnée à sa fertilité naturelle... les hommes dispersés parmi eux, observent, limitent leur industrie... " (Euvres complètes, P1. III, p. 135). Voir aussi pp. $136 ; 146 ; 152 ; 218$ et la note IV, p. 198.

17. Nous considérons dans cette cuvre le discours anthropologique ; nous n'ignorons pas qu'elle est aussi une œuvre politique, un exposé de philosophie de l'histoire et de psychologie sociale.

18. CEuvres complètes, P1., III, p. 124. 
désirent connaître la nature humaine, difficultés accrues par l'accumulation même des connaissances sur l'homme ${ }^{19}$, Rousseau revendique le seul mérite de présenter le véritable état de la question: «que mes lecteurs ne s'imaginent pas, dit-il, ... que j'ose me flatter d'avoir vu ce qui me paraît si difficile à voir. J'ai commencé quelques raisonnements ; $j$ 'ai hasardé quelques conjectures, moins dans l'espoir de résoudre la question que dans l'intention de l'éclaircir et de la réduire à son véritable état ${ }^{20}$ \%.

Fausse modestie, dira-t-on. L'explication serait certainement valable si l'auteur n'avait parlé sans cesse d'autres perspectives, d'autres approches qui permettraient de mieux connaître la nature de l'homme ${ }^{21}$. D'ailleurs, sa pensée anthropologique ne s'est pas limitée au seul Discours sur l'Inégalité; elle s'étale dans l'Émile ${ }^{22}$, et dans la Profession de foi du vicaire savoyard ${ }^{23}$.

Il ne s'agit nullement dans ces œuvres de simple répétition des thèses anthropologiques du second Discours. Si d'une œuvre à l'autre, le lecteur retrouve la même opposition entre la nature et l'histoire, la même affirmation des principes de la liberté, de l'égalité et de la bonté, le même respect du principe de développement des choses humaines, il perçoit aisément les différences, voire même les contradictions. N'a-t-il pas, en effet, décrit Émile comme l'homme naturel vivant en société ${ }^{24}$ ? Et n'a-t-il pas invité

19. «Ce qu'il y a de plus cruel encore, c'est que tous les progrès de l'espèce humaine l'éloignant sans cesse de son état primitif, plus nous accumulons de nouvelles connaissances, et plus nous nous ôtons les moyens d'acquérir la plus importante de toutes, et que c'est en un sens à force d'étudier l'homme que nous nous sommes mis hors d'état de le connaître ». (Euvres complètes, P1., III, P. 122-123).

20. Ibid., p. 123.

21. «Supposons un Montesquieu, un Buffon..., nous verrions nous-mêmes sortir un monde nouveau de dessous leur plume, et nous apprendrions ainsi à connaître le nôtre..." (Ibid., p. 213-214).

22. Parlant de son traité d'éducation, Rousseau écrit à un de ses correspondants : “C'est un ouvrage assez philosophique sur ce principe avancé par l'auteur dans d'autres écrits que l'homme est naturellement bon" (Correspondance complète, établie par Ralph Alexander LeIGH, Droz, Genève, lettre no 3564 à Philibert Cramer le 13 octobre 1764, tome XXI, p. 248). Voir aussi Robert DeRATHÉ, «L'homme selon Rousseau » dans Études sur le Contrat social, actes des journées d'études tenues à Dijon les 3-4-5 et 6 mai 1962, les Belles-Lettres, Paris, 1964, pp. 213-217.

23. «Il faut du temps, dit le vicaire à son interlocuteur, pour vous exposer tout ce que je pense sur le sort de l'homme et sur le vrai prix de la vie " (CEuvres complètes, P1. IV, P. 565).

24. "Il y a bien de la différence entre l'homme naturel vivant dans l'état de nature et I'homme naturel dans l'érat de société » (EEuvres complètes, P1. IV, p. 483). 
ses lecteurs à bien distinguer Émile de l'homme sauvage ${ }^{25}$ ? Mais s'il en est ainsi, on est autorisé à dire que les perspectives où se placent les études anthropologiques du second Discours et de l'Émile ne sont pas les mêmes, que chacune de ces œuvres offre la vision d'une dimension particulière de la condition humaine.

La première adopte la perspective de l'histoire de l'homme en tant qu'espèce. L'état de nature n'est certes pas un état historique, par lequel aurait débuté l'humanité ${ }^{26}$. On ne peut pas cependant ignorer la volonté de l'auteur de porter son regard au tout début de l'histoire des sociétés humaines afin de mieux comprendre l'origine et le fondement de l'inégalité parmi les hommes. «Ô homme, liton dans l'Exorde, de quelque contrée que tu sois, quelles que soient tes opinions, écoute : voici ton histoire... Les temps dont je vais parler sont bien éloignés : combien tu as changé de ce que tu étais ! C'est pour ainsi dire la vie de ton espèce que je te vais décrire... » ${ }^{27}$. Ce que Rousseau a trouvé en ce degré zéro de l'histoire, c'est une humanité encore immergée dans l'animaliré : «En le (l'homme) considérant... tel qu'il a dû sortir des mains de la nature, je vois un animal moins fort que les uns, moins agile que les autres, mais à tout prendre, organisé le plus avantageusement de tous... ${ }^{28}$.

Si cette condition animale se situe dans les temps éloignés, trop éloignés pour que l'idée d'une histoire humaine en rapport avec ces temps ait quelque sens, et si l'entrée dans l'histoire signifie pour les hommes le commencement d'un processus de dénaturation, le naturel est ce qui ne se retrouve plus et toute société, si primitive soit-elle, est déjà dépravée. Rousseau a, à plusieurs reprises, insisté sur l'impossibilité de retrouver l'état de nature 29.

25. "Il ne faut pas confondre ce qui est naturel à l'état sauvage et ce qui est narurel à l'état civil» (lbid., p. 764).

26. Il s'agit, nous dit Rousseau, de «bien connaitre un état qui n'existe plus, qui n'a peutêtre point existé, qui probablement n'existera jamais» (Ibid., p. 123). Pour une interprétation contraire, voir Marc-Florea PLATTNER, Rousseau's State of Nature, Northern Illinois University Press, De Kalb, 1979.

27. Euvres complètes, Pl., III, p. 133.

28. Ibid., p. 134-135. Voir aussi p. 162-163.

29. «Mais la nature humaine ne rétrograde pas et jamais on ne remonte vers les temps d'innocence er d'éga lité quand une fois on s'en est éloigné ; c'est encore un des principes sur lesquels il a le plus insisté. » (Dialogue troisième, Euvres complètes, P1., I, p. 935). Voir aussi O.C., P1. III, p.207. 
Une telle impossibiliré ne signifie rien de moins que l'impossibilité de toute tentative de régénérer la société. Aussi, Rousseau n'a jamais pensé à proposer la solution révolutionnaire aux grands États ; son objet est d' «arrêter s'il était possible le progrès de ceux (des États) dont la petitesse et la situation ont préservés d'une marche aussi rapide vers la perfection de la société et vers la détérioration de l'espèce ${ }^{30}$.

Mais si la préservation du naturel est chose impossible au niveau de la société, elle ne l'est pas au niveau de l'individu, car chez ce dernier le naturel existe. Tout n'est-il pas bien, sortant des mains de l'auteur des choses? La tâche qui s'impose donc à celui qui souhaite voir la nature préservée, c'est de bien définir le naturel par rapport à tout ce qui vient de la société. Ce projet est, à vrai dire, conçu et présenté dans le Discours sur l'Inégalité. Rousseau n'a-t-il pas en effet déclaré que la première tâche à entreprendre est de séparer l'originaire et l'artificiel dans «la nature actuelle de l'homme ${ }^{31}$. Le second Discours ne réalise pas cette tâche, puisque l'auteur fait intervenir dans sa recherche des " paramètres temporels et collectifs ». Nous avons vu que Rousseau attache l'idée d'une expérimentation au projet de dissocier le naturel du social. Quelle place accorde-t-il à cette idée dans les œuvres postérieures au Discours?

Le manuscrit Favre - qui, on le sait, donne la première version de l'Émile - fournit un indice révélateur. Au bas d'un des feuillets de ce manuscrit, on peut lire le texte suivant:

"Si l'homme a été constitué par son auteur tel qu'il devait être, ce qu'il tient de la nature vaut mieux que ce qu'il tient de ses semblables. Ce préjugé paraît légitime, c'est à l'expérience à le confirmer ou à le détruire. Mais comment faire cette expérience? J'ai souvent réfléchi sur la méthode qu'il faudrait employer pour faire cette expérience. Il ne serait peut-être pas tout à fait inutile de chercher les moyens. Mais quels moyens de faire cette expérience? Mettons-nous à la place de celui qui voudrait la tenter et cherchons ${ }^{32}$ \%.

Ainsi, Rousseau n'a pas abandonné l'idée d'une connaissance expérimentale de la nature humaine; on peut voir aussi par là que

30. Dialogue troisième, Euvres complètes, P1., I, p. 935

31. Guvres complètes, P1., III, p. 123. Nous soulignons.

32. Euvres complètes, P1., IV, P. 1269, note a de la p. 59 
l'Émile est d'intention anthropologique ${ }^{33}$, quoiqu'il soit en même temps un traité d'éducation et de «droit politique». Comment se réalise cette connaissance expérimentale? Rousseau est trop conscient des difficultés de la réalisation d'une telle connaissance pour vouloir jouer les "Aristotes et les Plines de son siècle » ${ }^{34}$. De fait, sa recherche s'est présentée sous la forme de la relation d'une expérience imaginaire: "J'ai... pris le parti, écrit Rousseau, de me donner un élève imaginaire, de me supposer l'âge, la santé, les connaissances, et tous les talents convenables pour travailler à son éducation... ${ }^{35}$. Une telle approche est commune à plusieurs penseurs de l'époque. On la voit réalisée, par exemple, chez Buffon, que Rousseau considère comme l'un de ses maîtres. ${ }^{36}$

Mais à la différence de ces penseurs qui pratiquent ce que Diderot appelle une «espèce d'anatomie métaphysique », l'auteur de l'Émile réalise son expérience sous la forme d'une expérience imaginaire de l'éducation : le traité d'anthropologie qu'il construit est aussi un traité d'éducation. Comment s'explique le rapport de ces deux projets? Rousseau nous dit qu'il se donne «un élève imaginaire » : pourquoi un élève et non pas, comme chez Buffon, le «premier homme au moment de la création »? Pour Martin Rang, il n'est pas possible de trouver une réponse satisfaisante à toutes ces questions. Le choix de Rousseau, selon ce commentateur, «demeurera éternellement le secret de son esprit créateur ». ${ }^{37} \mathrm{Il}$ ne nous semble pas qu'on doive se résigner à cette solution

33. Cette intention est affirmée par Rousseau dans sa correspondance comme dans ses écrits autobiographiques. À un de ses correspondants, qui trouve le projet pédagogique de l'Émile irréalisable, il s'explique : "Vous dites très bien qu'il est impossible de faire un Émile. Mais je ne puis croire que vous preniez le livre qui porte ce nom pour un vrai traité d'éducation. C'est un ouvrage assez philosophique sur ce principe avancé par l'auteur dans d'autres écrits que l'homme est naturellement bon "; (lectre déjà cirée à la note 22); "L'Émile, en parriculier, ce livre tant lu, si peu entendu et si mal apprécié, n'est qu'un traité de la bonté originelle de l'homme " (Dialogue troisième, O.C., P1., I, p. 934).

34. Sur ces difficulités, voir Eavres complètes, P1. III, p. 123-124.

35. Émile, Euvres complètes, P1., IV, p. 264.

36. Voir Buffon, De l'Homme, introduction de Michèle Ducher, Fr. Maspéro, 1971; "J'imagine donc un homme tel qu'on peut croire qu'il était le premier homme au moment de la création..." p. 214.

37. Martin RanG cité par Günther Buck, "La place systématique de l'Émile dans l'œuvre de Rousseau" dans Revue de théologie et de pbilosopbie, 110 (1978), p. 366. 
d'impuissance. Il nous semble au contraire que ce choix s'explique par le principe fondamental de sa pensée, par sa vision critique de la société et par sa conception de l'éducation.

Si dans la Lettre à Christophe de Beaumont, dans les Dialogues et dans sa correspondance, Rousseau parle du principe de la bonté naturelle comme d'un axiome, la note du Manuscrit Favre - que nous avons citée - le présente comme une hypothèse: " $\mathrm{Si}$ l'homme a été constitué par son auteur tel qu'il devait être, ce qu'il tient de la nature vaut mieux que ce qu'il tient de ses semblables. Ce préjugé paraît légitime, c'est à l'expérience à le confirmer ou à le détruire ». ${ }^{38}$ Quelle est la teneur de cette hypothèse?

Pour bien des commentateurs, la thèse de la bonté naturelle a été élaborée de façon graduelle. Cette vue contredit une déclaration de Rousseau dans les Dialogues, selon laquelle sa thèse est présente dans tous ses écrits, du Premier Discours jusqu'à l'Émile. Il y a sans doute des raisons pour ne pas voir dans cette déclaration une exagération de la part de son auteur, mais ce qui justifie en partie l'interprétation des commentateurs, c'est le fait que le principe de la bonté naturelle n'est développé que dans l'Émile. C'est, en effet, dans cet ouvrage que Rousseau donne à son principe une ampleur, une netteré et une consistance incontestables. L'Émile nous montre la bonté naturelle dans l'ordre physique aussi bien que dans l'ordre affectif, intellectuel et moral. Cette bonté est fragile: l'âme de l'enfant prend facilement de mauvais plis au moindre écart par rapport à l'ordre de la nature. Que, par suite d'une négligence ou d'un excès de soin, l'enfant ne connaisse pas le sentiment de l'amour maternel ou qu'il devienne vulnérable à l'adversité des choses, "nous voilà dès les premiers pas hors de la nature " ${ }^{39}$. De là la nécessité de s'occuper tôt de l'éducation de l'enfant : «Voulezvous, dit encore Rousseau, qu'il garde sa forme originelle? Conservez-la dès l'instant qu'il vient au monde. Sitôt qu'il naît, emparez-vous de lui, et ne le quittez plus qu'il ne soit homme: vous ne réussirez jamais sans cela " ${ }^{40}$. C'est cette idée d'une bonté

38. Guvres complètes, P1., IV, p. 1269.

39. Ibid., p. 259.

40. Ibid., p. 261 
positive, fragile et coextensive à toutes les dispositions de l'être humain qui est mise à l'épreuve dans l'expérience imaginaire de l'Émile.

Cetre mise à l'épreuve est nécessaire du moment que l'idée de bonté naturelle est contredite par l'expérience du mal. Ce que Rousseau voit dans le monde, c'est la déraison, le malheur, la servitude, les vices de toute espèce, la méchanceté des hommes. Du Premier Discours à l'Émile, la vision que l'auteur nous donne des hommes vivant en société est toujours sombre et pessimiste. Les hommes sont pervers..., déclare le Premier Discours ; ${ }^{41}$ le second affirme que ce que nous montre la société, c'est «la violence des hommes puissants et l'oppression des faibles $\gg .{ }^{42}$

Faudrait-il, pour lever cette contradiction, croire que les hommes sont méchants par nature, et adopter par exemple le dogme du péché originel ou la philosophie de Hobbes? Une telle solution heurterait trop ses convictions personnelles pour que Rousseau puisse l'accepter. Il lui faudra donc découvrir une autre voie pour résoudre la contradiction intolérable entre son principe et son expérience humaine.

C'est sa conception de l'éducation qui le met sur cette voie. Pour Rousseau, l'éducation n'est jamais une activité limitée à un certain âge de l'individu humain, à un certain domaine et impliquant seulement des relations interpersonnelles. Elle est le processus par lequel l'être humain change et diffère de ce qu'il est au moment de sa naissance, quels qu'en soient l'agent responsable et l'effet: "Tout ce que nous n'avons pas à notre naissance, écrit Rousseau, et dont nous avons besoin étant grands nous est donné par l'éducation. Cette éducation nous vient de la nature, ou des hommes, ou des choses $\gg 43$.

C'est cette notion de l'éducation qui a permis à Rousseau de concilier les deux vérités qui paraissent inconciliables, toutes deux étant également évidentes à ses yeux. Du fait de la puissance de

41. OEuvres complètes, P1., III, p. 15

42. Ibid., p. 127; “... je ne vis plus qu'erreur et folie dans la doctrine de nos sages, qu'oppression et misère dans notre ordre social" (Confessions, OEuvres complètes, P1., I, p. 416).

43. Guvres complètes, P1., IV, p. 247. Voir aussi O.C., P1., IV, p. 62 ; p. 252. 
l'éducation, de la malléabilité de l'être humain et de la fragilité de la bonté naturelle, il n'y aurait pas de contradiction à affirmer d'une part que l'homme est essentiellement bon et d'autre part, que l'homme est méchant: cette méchanceté est l'effet d'une mauvaise éducation; elle est l'aboutissement d'un devenir. La recherche anthropologique doit donc s'orienter vers la description de ce devenir, c'est-à-dire montrer les effets de l'éducation. C'est dans cet esprit qu'il nous faut comprendre les éclaircissements de Rousseau sur la substance de son livre : «Pour accorder ce principe (de la Bonté naturelle), écrit-il à Philibert Cramer - dans une lettre que nous avons citée - avec cette autre vérité non moins certaine que les hommes sont méchants, il fallait dans l'histoire du cœur humain montrer l'origine de tous les vices».

L'Émile s'attache à écrire l'histoire de la mauvaise éducation ou, pour reprendre les termes mêmes de Rousseau, «l'histoire du cœur humain». Il n'est donc pas étonnant de trouver l'Émile rempli d'observations sur l'enfance, sur les systèmes d'éducation, sur les pratiques courantes d'élever les enfants, de voir Rousseau souligner son attachement au réel et l'absence chez lui de tout esprit de système: "Ce qui me rend, dit-il, plus affirmatif et, je crois, plus excusable de l'être, c'est qu'au lieu de me livrer à l'esprit de système, je donne le moins qu'il est possible au raisonnement et ne me fie qu'à l'observation. Je ne me fonde point sur ce que j'ai imaginé, mais sur ce que j'ai vu " ${ }^{44}$.

Parallèlement à cette histoire de l'éducation dans les limites d'une vie humaine, Rousseau esquisse - selon ses mots - « un nouveau système d'éducation dont (il) offre le plan à l'examen des sages " ${ }^{45}$. L'Émile présente à la fois une histoire et une philosophie de l'éducation. La présence de cette dernière s'explique par la logique de l'enquête anthropologique, qui se réalise sous la forme que nous venons de définir : l'Émile ne peut décrire l'histoire réelle du cœur humain sans décrire en même temps l'histoire telle qu'elle aurait pu être si l'homme avait suivi «la marche de la nature», c'est-à-dire s'il avait reçu l'éducation naturelle. D'ailleurs, le projet d'établir un nouveau système d'éducation s'impose à celui qui a

44. Ibid, p. 550 ; voir aussi p. 348.

45. Lettres de la montagne, Ouvres completes, P1., III, p. 783. 
toujours le souci de rendre les hommes heureux ; et pour Rousseau, le bonheur n'est pas possible tant qu'on ne sait pas, dit-il, « distinguer la réalité de l'apparence, (et) l'homme de la nature de l'homme factice et fantastique que nos institutions et nos préjugés lui ont substitué » 46 .

Telles sont, nous semble-t-ii, les raisons qui permettent d'expliquer la fusion des deux projets anthropologique et pédagogique dans l'Émile, le fait qu' «un traité de la bonté originelle de l'homme » se présente en même temps comme «un traité de l'éducation».

Nous avons essayé précédemment de montrer que le projet de Rousseau dans l'Émile, c'est de chercher à connaître l'homme dans sa réalité essentielle par la voie d'une expérience imaginaire de l'éducation. La reconnaissance de cette intention fondamentale nous permet de comprendre pourquoi l'auteur désapprouve ceux de ses lecteurs qui considèrent l'Émile comme un traité de pédagogie, comme un recueil d'instructions à l'usage des parents. La cinquième des Lettres écrites de la montagne affirme que l'Émile n'a pas pour but d'offrir « une méthode pour les pères et les mères, à laquelle (l'auteur) n'a jamais songé ${ }^{47}$. Répondant à un certain monsieur Hangardt, qui se flatte d'avoir suivi scrupuleusement l'enseignement de l'Émile dans l'éducation de son fils, Rousseau a cette réplique: «Tant pis, monsieur, pour vous et pour votre fils, tant pis " ${ }^{48}$.

L'attitude de Rousseau comme les éclaircissements qu'il fournit à ses correspondants sur la signification première de son cuvre nous montrent à quel point se sont mépris ceux qui, pour avoir lu l'Émile comme un traité d'intention pédagogique, dénoncent les utopies et les absurdités de son soi-disant système d'éducation. L'auteur de la Critique de la Raison pure a fait cette remarque : «II n'est pas naturel qu'un homme passe la plus grande partie de sa vie à enseigner à un enfant comment il doit vivre lui-même un jour. Par conséquent, des précepteurs comme Jean-Jacques Rousseau

46. Dialogues, OEuvres complètes, P1., I, p. 728.

47. Cuvres complètes, P1,, III, p. 783.

48. Jean-Jacques Rousseau, Émile, Introduction par François et Pierre Richard, Paris, Grenier, 1954, p. XXXVIII. 
sont pure invention " ${ }^{49}$. Cette critique serait pertinente si l'intention de Rousseau était de proposer Émile et son précepteur comme modèles de relations pédagogiques. Le caractère délibérément impersonnel, schématique de ces deux personnages, leur isolement par rapport à toute société conviennent mal à des modèles. Par contre, ils conviennent parfaitement à des symboles. Émile, nous rappelle Rousseau, est «l'homme abstrait, l'homme exposé à tous les accidents de la vie humaine » ${ }^{\circ 0}$. Quant au précepteur, il est doué de toutes les qualités, de tous les talents convenables pour travailler à l'éducation d'Émile. C'est dire qu'Émile et son précepteur représentent la nature même de l'homme, l'un dans son développement, l'autre dans son état d'achèvement. «Émile et Mentor, écrit excellemment Jeanine Eon, n'entretiennent pas une relation interpersonnelle, ce sont des figures philosophiques. Mentor est l'être achevé : l'éducation qui le fit pleinement homme est supposée, il n'évoluera pas au cours de la démarche, il représente la raison, la conscience. Émile en revanche est pur devenir... ${ }^{51}$.

Les pédagogues dénoncent l'irréalisme du système d'éducation de Rousseau; de leur côté, les psychologues relèvent le caractère spéculatif et artificiel de sa psychologie. Cette critique est formulée par Arthur Chuquet en ces termes: "Émile vit d'abord par les sens, puis par l'intelligence, puis par la raison. Mais peut-on ainsi diviser l'existence et la découper par tranches? Rousseau oublie que la nature développe à la fois toutes les forces de l'âme » ${ }^{52}$. Dans le même esprit, Jean-Louis Lecercle déplore que Rousseau ait méconnu la dimension affective de l'enfance: «Ce qui enlève le plus de vie à Émile, écrit ce commentateur, c'est la rigueur implacable avec laquelle lui est refusée toute affectivité avant la

49. KANT cité par Jean Ferrari, Les sources françaises de la philosopbie de Kant, Paris, Klincksieck, 1979, p. 197, note 22. « De telles conditions sont requises pour l'éducation d'Émile, qu'il n'y aurait, dans tout le royaume de France, quelques centaines d'enfants qui puissent recevoir une éducation de cette sorte " (Jules Lemaitre cité par Alexis Philonenko, J.J. Rousseau et la pensée du malheur, t. 3, Vrin, 1984, p. 98).

50. Guvres complètes, P1., IV, p. 252.

51. Jeanine EON, «Émile, ou le roman de la nature humaine » dans Jean-Jacques Rousseau et la crise contemporaine de la conscience, colloque international du deuxième centenaire de la mort de J.J. Rousseau, Chantilly 5-8 septembre 1980, Beauchesne, Paris, 1980, p. 136.

52. Arthur ChuQuet, Jean-Jacques Rousseau, Hachette, Paris, 8e édition, 1929, p. 122. 
quinzième année ${ }^{53}$. Toutes ces critiques seraient certainement valables si l'auteur de l'Émile avait voulu faire œuvre de psychologue, s'il s'était proposé de décrire le développement psychologique de l'être humain. Or, nous l'avons vu, Rousseau a suivi les penseurs de l'époque pour appliquer la méthode de l'expérience imaginaire, méthode qui l'oblige à opérer une dissociation des diverses structures psychiques en vue de mieux «démêler ce qu'il y a d'originaire et d'artificiel» en chacune d'elles. Il s'agit pour Rousseau de faire œuvre de philosophe en effectuant une analyse génétique en respectant un ordre qui accorde la primauté ontologique à la sensibilité. C'est seulement au livre IV que Rousseau étudie la structure de l'affectivité ; pourtant, on peut déjà trouver dans les trois premiers livres des notations sur la vie affective de l'enfant, par exemple sur son attachement à sa nourrice ${ }^{54}$. Ce fait est significatif : le découpage de l'existence dans l'Émile répond à une exigence méthodologique; il n'est nullement l'effet de la méconnaissance de la complexité du psychisme. Que Rousseau n'ait pas l'intention de se placer sur le plan de la psychologie empirique se voit dans la lettre à Philibert Cramer, où il revendique pour l'Émile le statut d'un ouvrage " philosophique ». ( C C'est un ouvrage assez philosophique sur ce principe avancé par l'auteur dans d'autres écrits que l'homme est naturellement bon ") "5. Quand on connaît la critique que Rousseau fait de la philosophie et des philosophes, on peut voir combien est significative une telle revendication.

$$
* * * *
$$

Avec l'Émile, Rousseau a résolu à sa façon le problème qu'il s'était posé dans la préface du Discours sur l'Inégalité. L'Émile est bien un traité d'anthropologie ou, pour reprendre les termes mêmes de l'auteur, une étude de la condition humaine ${ }^{56}$. C'est en tenant compte de cette intention et de l'approche réalisée dans l'Émile que l'on peut rendre raison de certaines de ses particularités

53. Voir note 22 plus haut.

54. Jean-Louis LeCERCle, Rousseau et l'art du roman, A. Colin, Paris, 1969, p. 324.

55. Voir CEuvres complètes, P1., IV, pp. 257;259; 492.

56. “Notre véritable étude est celle de la condition humaine 》 (CEuvres complètes, P1., IV, p. 252). 
et par là même percevoir la non-pertinence de certaines critiques qui lui sont habituellement adressées. Mais dire que l'Émile représente la solution du problème de l'anthropologie rousseauiste, c'est s'obliger à reconnaître la particularité, donc les limites de l'anthropologie du Second Discours. À la lumière des remarques précédentes, il nous semble permis d'affirmer que, dans la recherche passionnée de la nature humaine de Rousseau, ce Discours ne représente qu'un aspect et n'offre qu'une perspective. Déjà dans ce discours, il a suggéré à ses lecteurs de ne pas prendre cette cuvre pour son discours anthropologique fondamental et définitif. Ce n'est pas par fausse modestie qu'il déclare dans la Préface qu'il a seulement «commencé quelques raisonnements (et) hasardé quelques conjectures ». ${ }^{57}$ C'est dans le même esprit qu'il fonde, sur les recherches que pourraient faire des hommes compétents, l'espoir de voir la connaissance de l'homme atteindre à plus de vérité: “Supposons, écrit Rousseau dans la note X, un Montesquieu, un Buffon, un Diderot, un Duclos, un d'Alembert, un Condillac, ou des hommes de cette trempe voyageant pour instruire leurs compatriotes, observant et décrivant comme ils savent faire...; supposons que ces nouveaux Hercules, de retour de ces courses mémorables, fissent ensuite à loisir l'histoire naturelle, morale et politique de ce qu'ils auraient vu, nous verrions nous-mêmes sortir un monde nouveau de dessous leur plume, et nous apprendrions ainsi à connaître le nôtre... ${ }^{58}$.

S'il est donc vrai que le Discours sur l'Inégalité, en tant que discours anthropologique, a ses limites, on devrait se défaire de cette vue - trop longtemps admise - selon laquelle l'homme naturel de Rousseau est ce Sauvage «... errant dans les forêts, sans industries, sans parole, sans domicile, sans guerre et sans liaison, sans nul besoin de ses semblables... " ${ }^{59}$. On devrait s'interdire de croire que la conception de l'homme de ce Discours puisse servir de base à toute la philosophie de l'auteur de l'Émile.

Département de philosophie

Université d'Ottawa

57. Guvres complètes, P1., III, P. 123.

58. Ibid., p. 213-214.

59. Ibid., p. 159-160. 